\title{
ENRIQUECIMIENTO TIPO III: EXPERIENCIAS URUGUAYAS EN LA ATENCIÓN DE ESTUDIANTES CON ALTAS HABILIDADES/ SUPERDOTACIÓN
}

\section{TYPE III ENRICHMENT: \\ URUGUAYAN EXPERIENCES IN THE ATTENTION OF STUDENTS WITH HIGH ABILITY/GIFTEDNESS}

\author{
Mag. Karen Bendelman ${ }^{3}$ \\ Mag. Natalia Colino 4 \\ Mag. Leticia Arbelo 5
}

\section{RESUMEN}

Esta investigación fue realizada en el marco de las primeras formaciones en el área de Altas Habilidades/ Superdotación para docentes a nivel nacional a través del Ministerio de Educación y Cultura. En esta formación participaron 27 docentes, de los cuales se seleccionaron dos experiencias de su trabajo final: un estudiante de educación primaria que concurre a una escuela rural en el interior del país y un estudiante de secundaria que concurre a una institución privada de la capital del país. El objetivo general fue analizar cómo los docentes pusieron en práctica actividades de “Enriquecimiento Tipo III" (Renzulli \& Reis, 1997) y cómo cambió la actitud de los estudiantes a partir de la incorporación de estas experiencias en el aula. Los resultados muestran estudiantes motivados, productores de conocimiento (no consumidores), lo que lleva al aprendizaje en el aula a ser más desafiante y atrayente para todos los alumnos.

Palabras claves: educación diferenciada, altas habilidades/superdotación, enriquecimiento tipo III.

\section{ABSTRACT}

This research was conducted in the framework of the first courses in the area of High Ability/Giftedness for teachers at the national level through the Ministry of Education and Culture. 27 teachers participated in this training, of which two experiences of their final work were selected: a primary school student who attends a rural school and a high school student who attends a private institution in the capital of the country. The general objective was to analyze how teachers implement "Type III Enrichment" activities (Renzulli \& Reis, 1997) and how the attitude of the students changed by incorporating these experiences in the class-

3 Psicopedagoga, Magíster en Psicología Educativa con énfasis en AH/SD, Universidad de Connecticut, EEUU. Delegada por Uruguay ante el Consejo Mundial de Superdotación (WCGTC) y miembro de la Asociación Norteamericana de Superdotación (NAGC). Asesora de AH/SD para gobiernos internacionales. Autora de varios artículos y tres libros sobre AH/SD. - karen@sobredotacion.com

4 Profesora de Matemática, Magíster en Educación. Doctoranda en Psicología con énfasis en Altas Habilidades. Referente de Cooperación Internacional en el Consejo de Formación en Educación. Entrenadora olímpica en Matemática y referente de altas habilidades/superdotación en instituciones educativas. nataliacolino@outlook.com

5 Maestra de Educación Común. Magíster en Educación. leticiaarbelomarrero@gmail.com 
room. The results show motivated students, producers of knowledge (rather than consumers), which leads to more challenging and attractive learning for all students.

Keywords: differentiated education, high ability/giftedness,type III enrichment.

\section{MARCO TEÓRICO}

Vivimos en la sociedad del conocimiento, pero nuestra educación se quedó en la revolución industrial. Se pone énfasis en la memorización y la repetición, en lugar de la creatividad y la comprensión. El currículo educativo es inflexible, y asume que todos los estudiantes tienen los mismos intereses y necesidades, las mismas fortalezas y debilidades.

La agenda de educación al 2030 pone especial énfasis en el derecho a recibir una educación inclusiva y de calidad durante toda la vida (UNESCO, 2017) y en este sentido, los educadores debemos reinventar nuestra gestión en el aula para mejorar los procesos de enseñanza-aprendizaje y obtener resultados que acompasen el desarrollo de la sociedad y la economía. La construcción de significados y nuevos conocimientos a partir del traslado de las propuestas curriculares oficiales a la práctica, ocupa un rol fundamental en la gestión en el aula (Villalobos, 2011). Esta construcción no será efectiva sin la impartición de una instrucción diferenciada a nuestros estudiantes.

¿Qué es realmente la diferenciación? La diferenciación es un proceso continuo mediante el cual, a partir del conocimiento de los intereses y las necesidades educativas especiales (a nivel académico, cognitivo, social y emocional) de los estudiantes, el docente va adaptando su rol en la clase y guiando la enseñanza con diferentes estrategias (Tomlinson, 1999). ¿Por qué debemos diferenciar? Los estudiantes aprenden sólo si se trabaja en su zona de desarrollo próximo, si existe un equilibrio entre sus habilidades y el nivel de desafío propuesto. Utilizar estrategias de diferenciación aumenta la probabilidad que el estudiante despliegue al máximo su potencial (Zmood, 2014). La instrucción diferenciada puede lograrse a partir de un currículo diferenciado, ya sea modificando los contenidos curriculares, es decir, lo que debe ser enseñado y aprendido, o añadiendo cambios en la transposición didáctica que refiere a cómo el contenido es explorado o desarrollado (ACARA, 2013).

Asimismo, Maker y Shiever (2010) añaden la importancia de adaptar el ambiente de aprendizaje, así como también el producto, para desarrollar un currículo cualitativamente diferente.

La instrucción diferenciada reconoce que algunos estudiantes dentro de una clase puedan beneficiarse particularmente con las adecuaciones mencionadas. Es el caso de los estudiantes que poseen dificultades de aprendizaje y/o altas habilidades/superdotación (AH/SD) en una o más áreas.

Uruguay ha avanzado en la atención diferenciada de estudiantes con dificultades de aprendizaje, pero aún carece de políticas educativas que reconozcan los derechos de los estudiantes con AH/SD. De acuerdo a estadísticas internacionales, Uruguay tendría alrededor de 300.000 personas con AH/SD, de las cuales 70.000 son estudiantes que se encuentran en nuestras aulas, sin embargo no existen servicios para poder atenderlos en centros educativos que atiendan sus necesidades educativas, sociales y emocionales.

Según Renzulli (1978) los estudiantes con AH/SD son aquellos que demuestran una habilidad muy por encima al promedio, altos niveles de creatividad y compromiso con la tarea (Fig.1). 


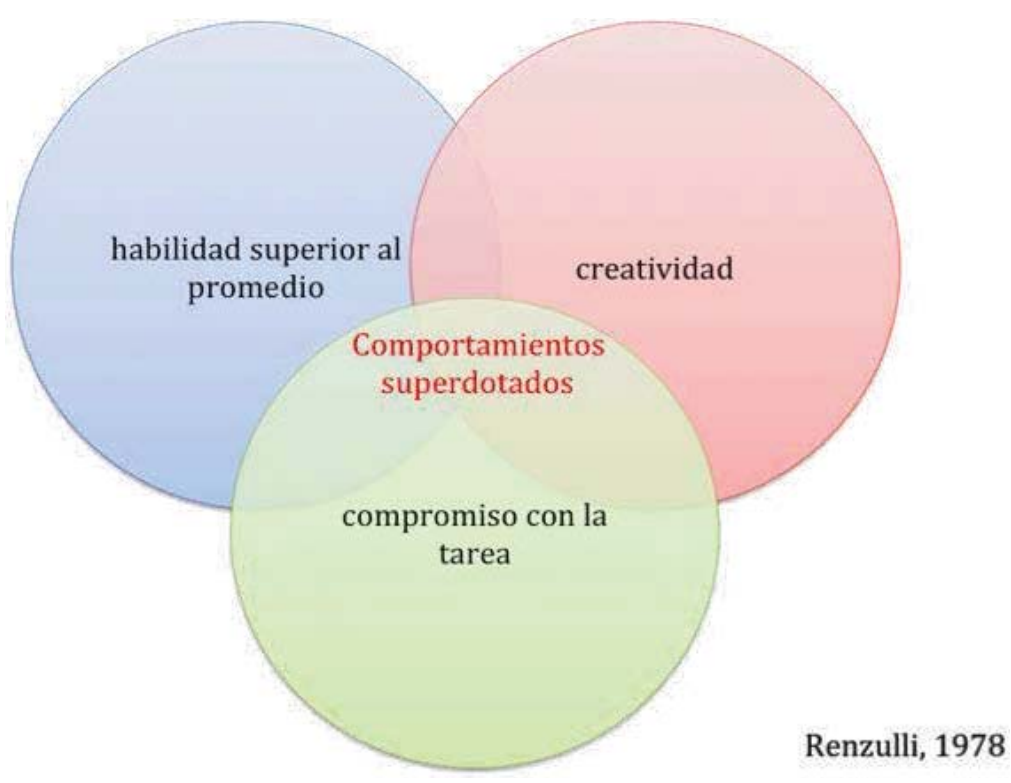

Figura 1: Concepto de los tres anillos de la superdotación Extraído de Bendelman (2012)

Para crear condiciones educativas favorables que potencien y estimulen la interacción entre los tres grupos de rasgos que componen el comportamiento superdotado, Renzulli creó el Modelo de Enriquecimiento Triárquico (Enrichment Triad Model, 1976).

El mejor modo de implementar el modelo es teniendo en cuenta los siguientes cuatro principios:

- Las experiencias deben estar alineadas con los estilos personales de aprendizaje de cada estudiante.

- El aprendizaje es más efectivo en la medida en que se disfruta.

- El aprendizaje es más significativo cuando se aprende en el contexto de problemas reales.

- El objetivo último del modelo es desarrollar estrategias de pensamiento, competencias y habilidades que le permitan al estudiante ser productivo-creativo. (Renzulli, 1995).

Existen tres tipos de enriquecimiento:

- El tipo I consiste en fomentar actividades generales exploratorias que introduzcan al alumno en áreas de conocimiento que no están contempladas en el currículo regular.

- El tipo II radica en actividades de entrenamiento para adquirir competencias.

- El tipo III lo constituyen proyectos o investigaciones dirigidas a resolver problemas reales. (Reis \& Renzulli, 1997).

Las actividades de enriquecimiento tipo III consisten en investigaciones individuales o en pequeños grupos, que pueden ser realizadas por estudiantes de cualquier edad, sobre temas reales de su interés; actividades de investigación y producciones artísticas en el cual el aprendiz asume el rol de investigador primario. "Una persona joven pensando, sintiendo y actuando como un profesional del área" (Renzulli \& Reis, 1997).

Los estudiantes son introducidos a la investigación como método para contestar sus preguntas. Son ellos mismos los que formulan sus preguntas y utilizan la investigación para encontrar las respuestas. Esto lleva a que se involucren en su propio aprendizaje, mientras que el docente es un facilitador de éste, ayudando a localizar recursos (Bendelman, 2012).

Es una oportunidad para el estudiante para poner en práctica sus intereses, creatividad y conocimiento. También es una manera que el aprendiz mida su compromiso con la tarea o actividad a realizar. La actividad debe incluir un proceso, un producto y contar con una audiencia para compartir los resultados. Uno de los objetivos es que el estudiante entienda el contenido y la metodología. El estudiante aprende cómo 
aprender por sí mismo, considerando aspectos como la organización, los tiempos, y la toma de decisiones durante la investigación. Es un aprendizaje personalizado que lleva al desarrollo de la autoconfianza, la motivación y la creatividad. Finalmente, el producto debe ser novedoso e impactar a la audiencia seleccionada. (Renzulli \& Reis, 1997)

El enriquecimiento tipo III es sensible a los intereses y los estilos de aprendizaje de los estudiantes y permite exhibir sus fortalezas y talentos. Este método estimula la resolución de problemas y la toma de decisiones mientras se trabaja en el proyecto. Los estudiantes adquieren conocimientos relevantes sobre el contenido del tema que eligieron estudiar y los ayuda a fortalecer las relaciones sociales mientras trabajan con otros. (Bendelman, 2012)

Los programas de enriquecimiento para estudiantes son considerados "laboratorios" de las escuelas del mundo porque presentan oportunidades reales para probar ideas nuevas y experimentar con soluciones potenciales a problemas que surgen.

En particular, los programas para estudiantes con altas habilidades han sido especialmente fértiles para la experimentación porque éstos no suelen estar guiados por el currículo prescripto ni por métodos tradicionales de enseñanza. Por el contrario, se focalizan en los conceptos en vez de en el aprendizaje de contenidos, en la utilización de un currículo interdisciplinar y aprendizaje por temas, portafolios de los estudiantes, evaluaciones basadas en el proceso y no en el producto, agrupamiento por interés y no por edad, y oportunidades para que los niños intercambien los roles tradicionales de aprendiz de lecciones y meros ejecutores, a roles desafiantes y demandantes que requieren aprendizaje práctico; investigaciones directas, aplicación de conocimiento y de habilidades de pensamiento aplicadas a problemas reales que surgen de la necesidad e interés del propio estudiante.

\section{FASES EN LA ELABORACIÓN DE UN PROYECTO}

Inspirado en la filosofía del "Project Approach" (Katz, Chard \& Kogen, 2014) decidimos dividir la organización de los ejemplos de Enriquecimiento tipo III que se describirán a continuación, en tres fases: En la primera fase del proyecto el/los estudiantes y el/la docente llevan a cabo discusiones para seleccionar y definir el tema a investigar (Katz, Chard \& Kogen, 2014). Dentro de esta fase inicial se comienzan a intercambiar conocimientos previos y definir preguntas a investigar. La segunda fase de un proyecto (o fase central) es tiempo de investigación y de búsqueda de respuesta a las preguntas iniciales; también el momento donde se va profundizando en el tema y se van planteando nuevas y más avanzadas preguntas. Finalmente, el proyecto culmina con la elaboración de un producto final, algo que sea novedoso, original y hecho por los propios estudiantes.

\section{EXPERIENCIAS DE ENRIQUECIMIENTO TIPO III EN URUGUAY}

En el año 2014, el Ministerio de Educación y Cultura decidió darle una oportunidad a la formación de docentes de todo el país sobre el tema Altas Habilidades/Superdotación (AH/SD), una materia no explorada en el país donde los propios docentes demandan capacitación, pero se carece de ella en formación docente y continuada. Esa fue la primer instancia que el país apostó por la capacitación en este tema; la formación ofrecida tenía una duración de 9 meses calendario (Marzo a Diciembre) donde se abordaron tópicos relativos al tema de AH/SD como: características de las personas con altas habilidades/superdotación (PAH/SD) mitos más comunes, identificación en el aula, aspectos socio-emocionales, opciones de atención dentro y fuera del aula, entre otros.

La formación impartida era teórico-práctico por lo que cada docente en ejercicio (requerimiento para ser aceptado/s) tenía instancias prácticas con sus estudiantes. Una de ellas consistía en la elaboración de un proyecto de trabajo por el/los estudiante/s sobre un tema de interés de acuerdo a los principios teóricos expuestos anteriormente (enriquecimiento tipo III). Como fue aclarado en la sección anterior, el docente fue un facilitador de este proceso de aprendizaje donde los propios alumnos fueron sus guías y motivadores para conocer sobre un tema en particular.

En este artículo queremos mostrar algunos ejemplos de experiencias de enriquecimiento mediante proyectos realizadas en escuelas y liceos del país durante el año 2016. En particular se discutirán detalles de la 
planeación, implementación y conclusiones de dos de ellas, bien distintas: la primera en un 4to grado de Educación Primaria en una escuela rural del Departamento de Lavalleja y otra en un 3er. año de secundaria en un liceo de Montevideo.

\section{EXPERIENCIA EN UNA ESCUELA RURAL}

\section{FASE I - ELECCIÓN DEL PROBLEMA A INVESTIGAR}

El tema escogido por la alumna, a quien llamaremos "María" para trabajar en el proyecto, fue en el área de las ciencias naturales y asociado a la disciplina biología. El tópico seleccionado fue elegido espontáneamente por la alumna y fue formulado al final de la investigación como: "Animales del bioma pradera: semejanzas y diferencias según sus cráneos". María cursaba 4to año de educación primaria en una escuela rural del Departamento de Lavalleja en el momento de la investigación.

La alumna comenzó la investigación desde el abordaje de los esqueletos de los animales en forma autónoma y libre. Debido a que es una niña con abundantes conocimientos acerca de la biología animal y sus características, a consecuencia de su gusto innato y la estimulación de su familia al respecto, ésta inició la profundización de sus conocimientos abordando diferentes especies y familias de animales. Comparó sus esqueletos mediante imágenes recopiladas en libros e internet, estableció semejanzas y diferencias notorias, afinando el tenor de estas al avanzar en el estudio del tema.

La docente se comportó como una guía y facilitadora de materiales y formatos de organización de los conocimientos abordados.

A medida que el tiempo transcurría se fue avanzando y profundizando en la temática. Así fue que el sistema locomotor de algunas especies fueron captando la atención de la alumna, quien se planteó la idea de construir una colección de cráneos de animales que habitan el bioma donde se encuentra ubicada la escuela y su hogar: la pradera natural. De esa forma comenzó a trabajar en su hogar, con ayuda de sus padres (en especial su padre, que es peón rural y transita a menudo lugares con gran variedad de fauna) en la recolección, procesamiento y acondicionamiento de diversos cráneos para su posterior comparación en el aula.

Paulatinamente fue presentando a la clase multiplicidad de cráneos de ejemplares tales como: vaca, caballo, oveja, zorro, zorrillo, mano pelada, perro, gato, lobo de río, venado de campo, guazubirá, ñandú, tatú, carpincho, nutria y algunos otros.

Al trascender la noticia que una alumna de la escuela estaba realizando esta investigación y recolección, fueron muchos los vecinos que colaboraron con el proyecto acercando cráneos para su posterior estudio escolar. Así fue como un capataz de estancia de un establecimiento cercano donó el cráneo de mano pelada y lobo de río; otro vecino realizó la donación de un cráneo de carpincho y de un cerdo jabalí; un alumno de la escuela colaboró con la donación de un cráneo de caballo y los huesos de las extremidades de un vacuno. Los cráneos llegaron en forma limpia, pero se procedió a su desinfección en formol durante 5 días para evitar cualquier patógeno y asegurar su durabilidad como pieza de estudio en el futuro.

Cabe destacar que las muestras recopiladas por la alumna fueron rescatadas de animales que fueron encontrados sin vida, por lo que algunos cráneos se encontraban muy destruidos o con faltantes debido a la intemperie y el pasaje del tiempo.

Todas las muestras se fueron observando y analizando en torno a sus semejanzas y diferencias según la familia y especie.

\section{FASE II - TRABAJO DE CAMPO}

Los proyectos se inspiran en el método científico, donde se hacen preguntas para investigar, se realizan predicciones y se utiliza la investigación para corroborar las hipótesis y encontrar las respuestas. Algunas de las preguntas iniciales planteadas por María fueron: 
1. ¿Qué huesos tienen en común los esqueletos de la mayoría de los animales? ¿Por qué?

2. ¿Cómo puedo identificar una especie mediante la observación de sus huesos, en especial del cráneo?

3. ¿Qué cosas podían variar entre cada animal, su especie y su tipo?

4. ¿Cuáles cráneos serían más abundantes en la naturaleza?

5. ¿Cuáles podrían durar más y por qué?

Mediante la recolección, análisis de cráneos y restos de partes de las extremidades de individuos de algunas especies se fue evolucionando en la temática de investigación y acrecentando los conocimientos empíricos. De esta forma la alumna fue obteniendo respuestas parciales a sus interrogantes y generando otras de mayor complejidad, construyendo hipótesis y refutando otras respecto a algunas características craneales de las especies estudiadas.

Hallazgos:

I) Los animales de buena visión poseen órbitas oculares muy pronunciadas

II) Los animales que posee buen olfato poseen cavidades nasales alargadas y profundas

III) Simplemente observando su dentadura es posible saber mucho acerca de los hábitos alimenticios y otras adaptaciones de los animales

IV) Los animales que poseen cráneos más frágiles, como el ñandú, que solo tiene la cavidad craneal compuesta por tejido óseo y todo lo demás compuesto de cartílagos; en su mayoría son aves.

V) Dependiendo de si el animal es carnívoro, herbívoro u omnívoro, varía la dentadura y la forma de la cabeza.

A medida que se avanzó en la investigación y se ahondó en el tema, de forma espontánea fueron surgiendo nuevas y más profundas preguntas. En el caso de María, algunos de ellas fueron:

44 ¿Qué relación tiene el tamaño de la cavidad craneana con la posible inteligencia del animal?

¿Por qué hay cráneos de especies abundantes, como por ejemplo de zorro, que no se encuentran con facilidad en la naturaleza?

¿Dependerá de la forma de la cabeza la inteligencia del animal? ¿Esto influirá en su forma de vida y de supervivencia?

Hallazgo:

VI) La mayoría de los herbívoros, fundamentalmente los de la pradera, tienen la cabeza alargada (caballo, vaca, venado). Muchas veces los carnívoros poseen cabezas achatadas, excepto en los caninos debido a que la mayoría de estos tienen el hocico largo.

En el transcurso de la investigación, la docente logró contactar un estudiante de veterinaria avanzada, ya que María sintió la necesidad de recabar información específica y detallada para poder responder a sus nuevas interrogantes. La estudiante es vecina de la escuela, y su encuentro con María fue muy fructífero para el proyecto. A raíz de este acercamiento, María expresó desear estudiar la carrera de veterinaria o biología para poder continuar con el aprendizaje de temas relativos a sus intereses.

\section{FASE III - PRODUCTO FINAL}

A medida que los tiempos de estudio fueron avanzando, surgió la necesidad de compartir conocimientos aprendidos y reformulados durante el proyecto con los demás integrantes del grupo-clase (multigrado). Para esto, María elaboró una exposición de cráneos (de una amplia variedad de especies) y algunas piezas del aparato locomotor (vaca y ñandú), con ayuda de un compañero de $6^{\circ}$ grado que voluntariamente brindó su colaboración. 
El cierre parcial del proyecto estuvo dado por una charla de la alumna a los integrantes del grupo multigrado de su escuela donde se explicó la metodología de trabajo y estudio, se expusieron algunas semejanzas y diferencias entre esqueletos de especies diferentes, culminando con la visita guiada a la exposición de cráneos y piezas óseas recopiladas durante el proyecto.

La modalidad de trabajo por proyecto resultó una estrategia muy amena y de gran interés para María.

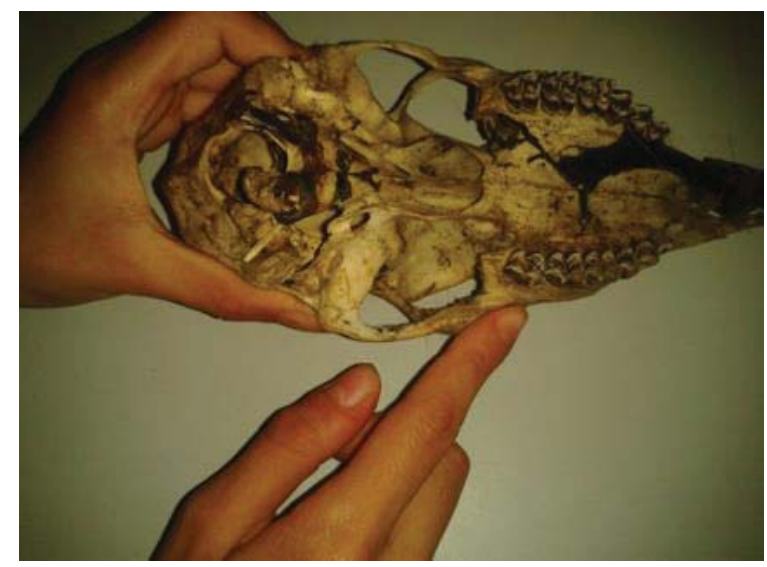

Figura 2: Exponiendo las características del cráneo de venado de campo

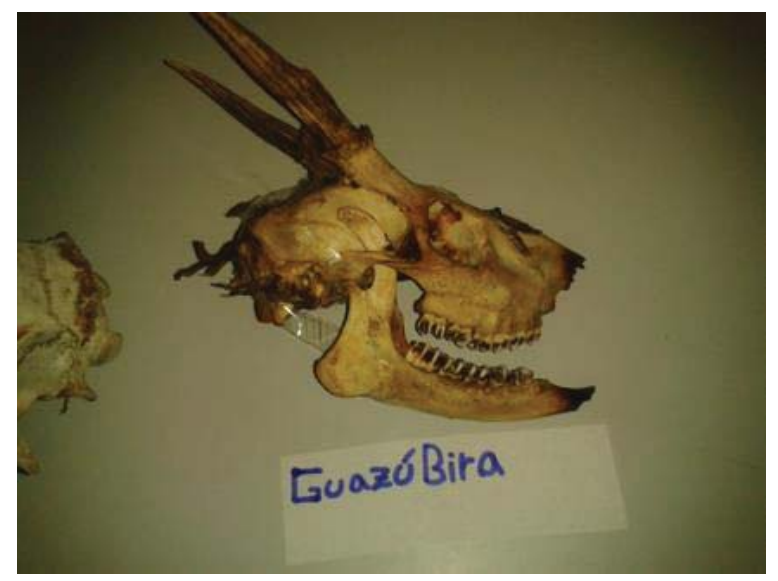

Figura 3: Cráneo de cérvido perteneciente a la exposición

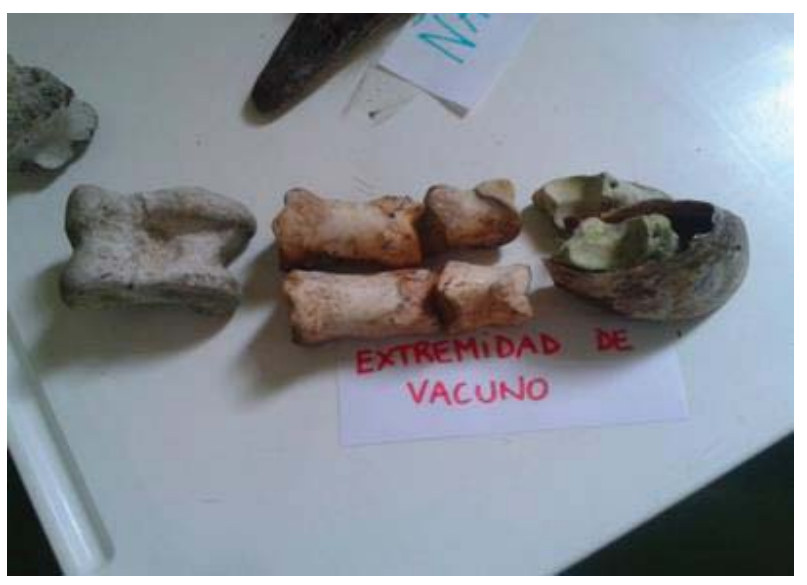

Figura 4: Piezas óseas de la extremidad de un vacuno 


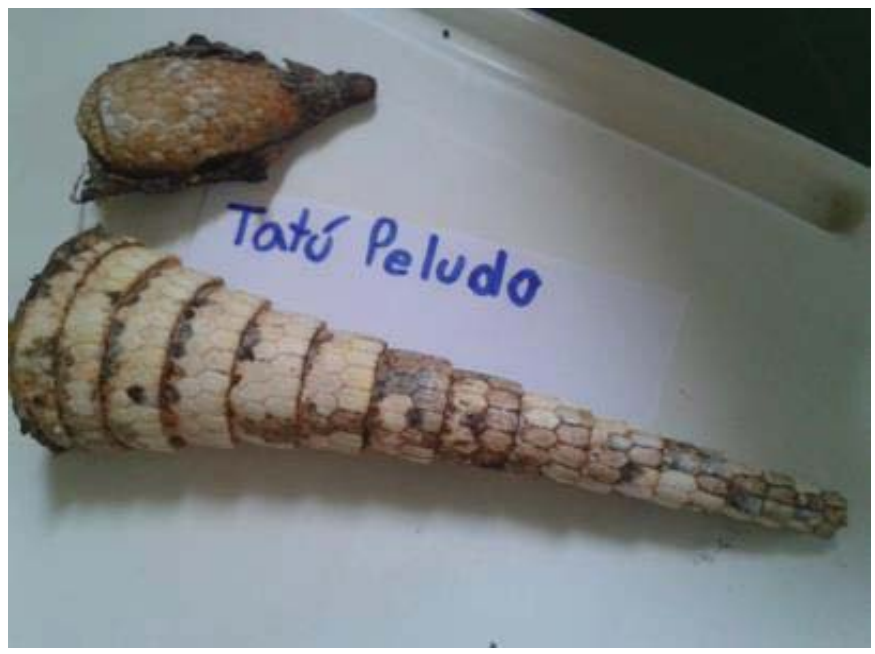

Figura 5: Cráneo y rabo de tatú peludo

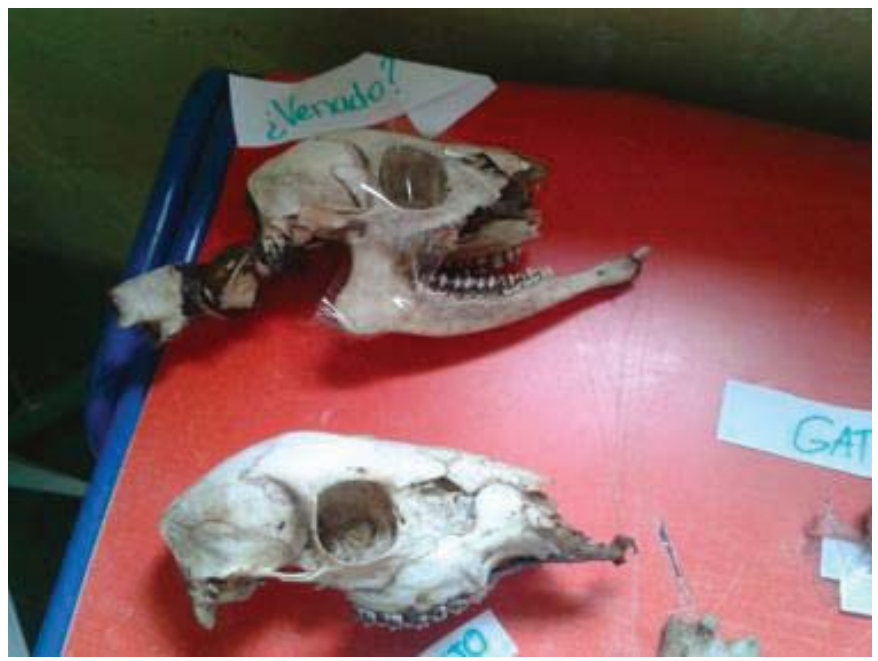

Figura 6: Cráneos de venado

Esta fotografía muestra la confirmación de una hipótesis de la alumna, quien al observar el cráneo de más arriba creía que este podía ser de venado de campo (considerando sus características). Días más tarde se encontró con otro cráneo similar en el campo, y al consultar con la estudiante de veterinaria, ésta confirmó que realmente estaban ante cráneos de venado. Hipótesis comprobada. 


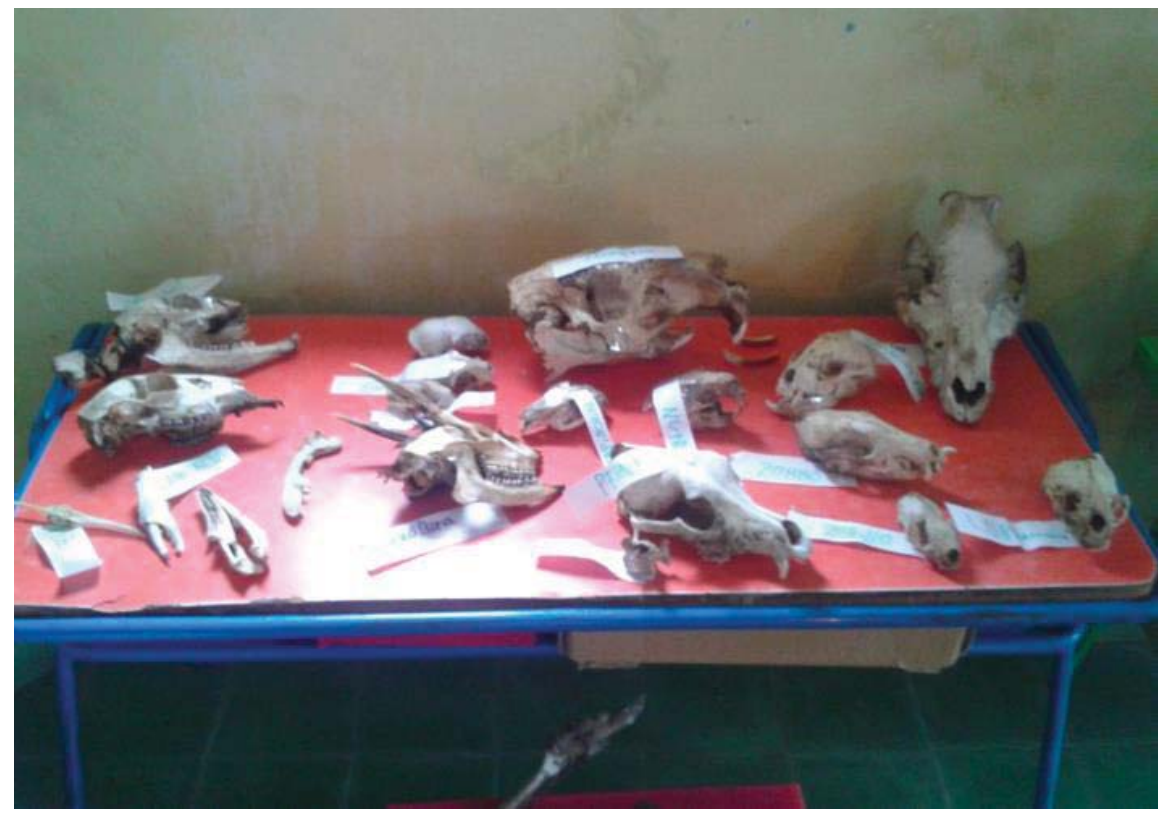

Figura 7: Parte de la exposición de piezas óseas elaborada por María

\section{EXPERIENCIA EN UN LICEO DE MONTEVIDEO}

\section{FASE I - ELECCIÓN DEL PROBLEMA A INVESTIGAR}

La tarea de enriquecimiento tipo III se propuso en un grupo de matemática de tercer año del Ciclo Básico en un liceo de Montevideo. En el marco de ir formando a los alumnos para el Bachillerato, se propuso a los estudiantes llevar a cabo un proyecto de investigación con foco en un tema de su interés. Se les indicó que el trabajo sería de carácter opcional, con calificación, que podría realizarse en equipos de libre elección con un máximo de cuatro integrantes, y que una vez culminado, tendrían la oportunidad de presentar lo investigado al resto de sus compañeros dentro del horario de clase.

Una vez presentada la propuesta de trabajo al grupo, uno de los estudiantes al que llamaremos Juan, no dudó en aceptar el desafío y se acercó a la docente para manifestar su interés. Posteriormente, decidió no afrontar solo esta tarea sino por el contrario, invitó a sus tres compañeros de banco con quienes trabaja habitualmente en la clase, a realizar juntos el proyecto. Cabe destacar que tanto Juan como los compañeros elegidos por él han sido identificados como alumnos con altas habilidades. Finalmente, se acordó con los cuatro integrantes del equipo, que por ser todos ellos alumnos del espacio de Olimpíadas de Matemática, se dedicaría al proyecto la primera parte de esa hora semanal con el fin de poder ser orientados en su trabajo. La elaboración del proyecto comprendió cinco encuentros.

\section{Primer encuentro}

Se invitó a los miembros del equipo a manifestar sus inquietudes, registrando en el pizarrón aquellos temas que les provocaba curiosidad, indicando el o los aspectos en los cuales les gustaría focalizarse. Una vez que los cuatro integrantes se habían expresado, se les solicitó que eligieran un sólo tema procurando que todos quedaran satisfechos con dicha elección. Luego de discutir acerca de lo interesante que sería investigar cada uno de los diez temas expuestos, acordaron enfocarse en el estudio de "La física detrás de un tug of war" $^{\prime \prime}$. La siguiente imagen sintetiza lo trabajado en este encuentro.

6 "Tug of war" o la cinchada de cuerda es un deporte que enfrenta a dos equipos, sometiéndolos a una prueba de fuerza. Cada equipo cincha de un extremo de la cuerda con el objetivo de acercar al equipo rival hasta que la marca más cercana a éste cruce la línea central. 


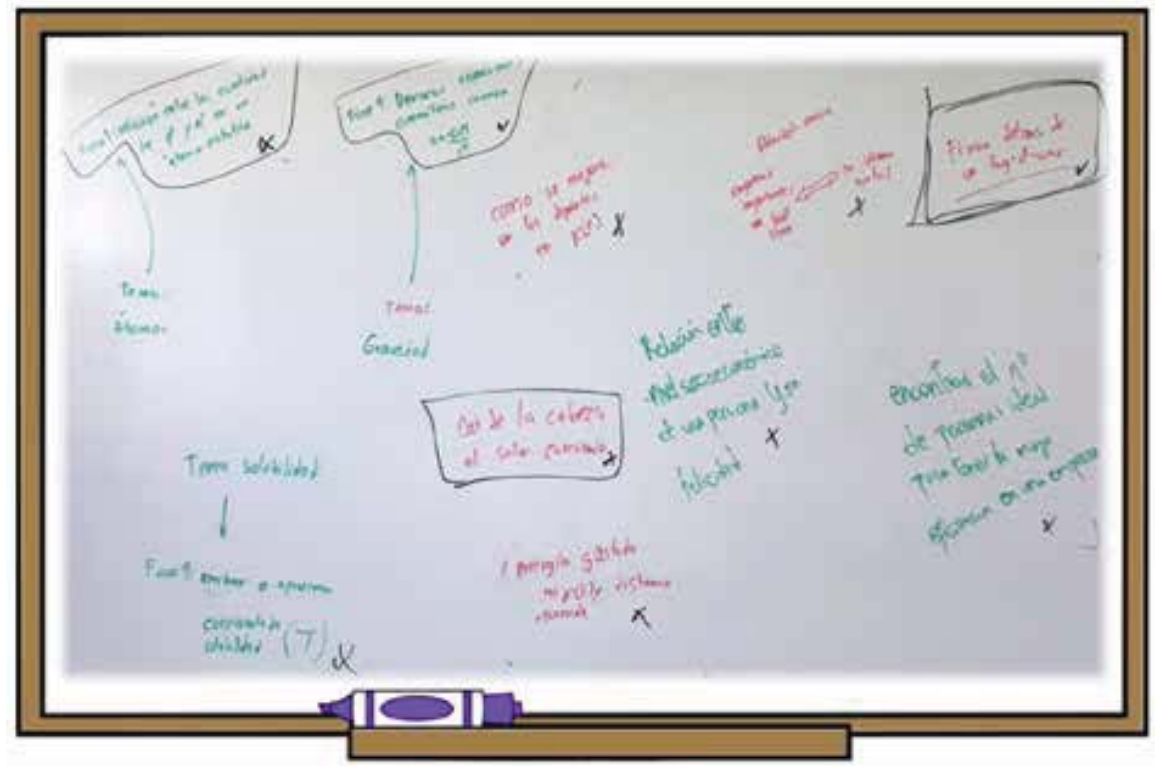

Figura 8: Posibles temas a investigar

\section{Segundo encuentro}

Se les propuso a los alumnos plasmar en el pizarrón un listado de los conocimientos previos que tenían, relacionados con el tema elegido, y que por lo tanto les serían de utilidad. Esto fue lo que escribieron:

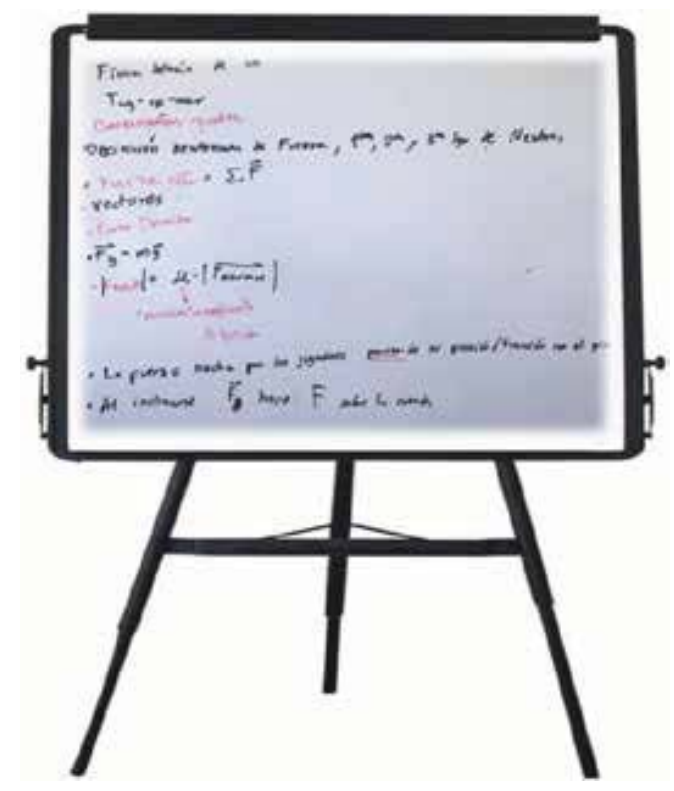

Figura 9: Exposición de conocimientos previos 


\section{Tercer encuentro}

En esta oportunidad, se le planteó como objetivo al equipo delimitar el problema. En primer lugar, se les solicitó que pensaran en varias preguntas asociadas a "La física detrás de un tug of war" que los motivara a investigar para conocer las respuestas. A continuación, se muestran sus inquietudes:

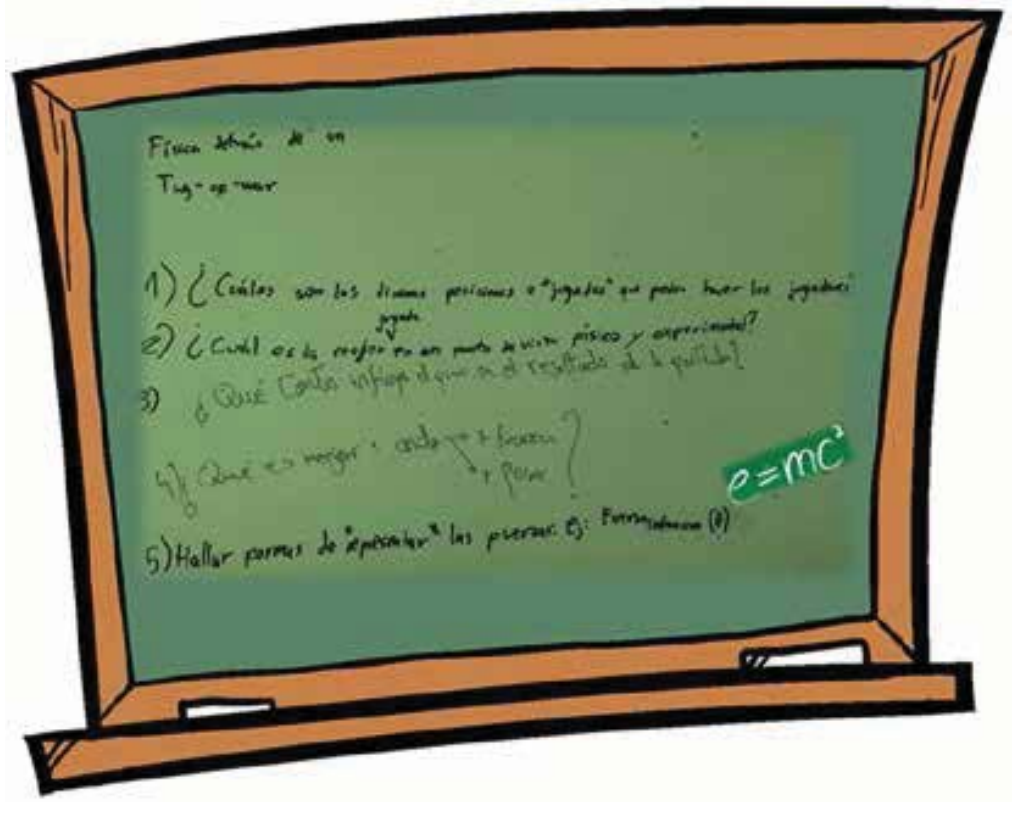

Figura 10: Posibles preguntas de investigación

En segundo lugar, debieron elegir sólo una de ellas y formularla como pregunta central del proyecto de investigación. Si bien eligieron la pregunta 1, se las ingeniaron y la reformularon de forma que abarcara a las preguntas 2 y 3 . Lo hicieron de esta manera: "¿Cuáles son las mejores estrategias para ganar un "tug of war"? ¿Cómo optimizar el trabajo de equipo?"

\section{Cuarto encuentro}

En esta etapa del proyecto, se les solicitó a los alumnos que expusieran sus hipótesis respecto a las respuestas que esperaban obtener en la pregunta central de investigación que se habían formulado. Se les explicó que a medida que avanzara su investigación podrían descartarlas o comprobarlas. Dado que el equipo no se ponía de acuerdo con sus creencias respecto al "tug of war" decidieron traer al salón una cuerda para comenzar a experimentar entre ellos y luego con el resto de los alumnos del espacio de Olimpíadas de Matemática que estaban allí presentes y que también se habían entusiasmado con el proyecto. Finalmente, arribaron a las siguientes hipótesis: 


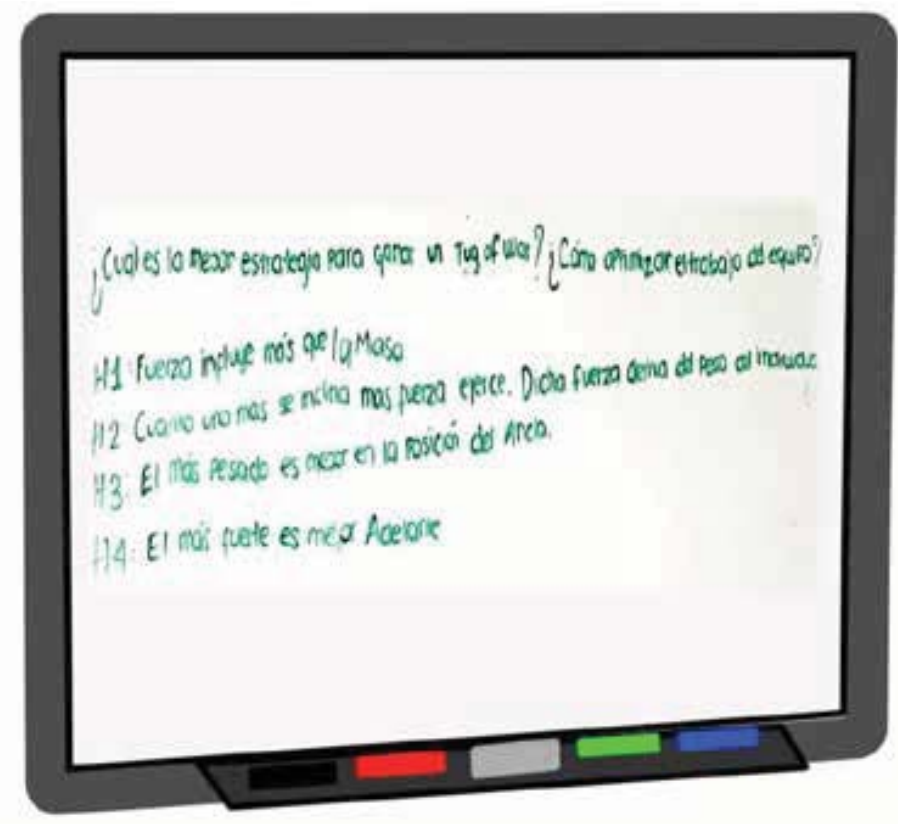

Figura 11: Formulación de hipótesis

\section{FASE II - TRABAJO DE CAMPO}

$50 \mid$

\section{Quinto encuentro}

Luego del cuarto encuentro, el equipo comenzó con su trabajo de campo y para ello decidió armar un grupo de "whats app" con la docente. A través de este medio se compartieron las fotos que habían sido tomadas del pizarrón con los resultados obtenidos en las diferentes etapas del proyecto, se guió la búsqueda de la bibliografía asociada con el tema de investigación y se fueron intercambiando los hallazgos encontrados. Asimismo, los miembros del equipo no dudaron en contactar a su profesor de Educación Física, a su docente de Ciencias y a la bibliotecóloga del liceo para recabar mayor información para su proyecto. Sin embargo, no se obtuvieron datos profundos de esta fase del proceso. Al tratarse de adolescentes curiosos, adoptaron un estilo de aprendizaje autoguiado y autónomo.

Adicionalmente, se les sugirió a los cuatro integrantes crear un documento en "google drive" para ir redactando el trabajo final e ir ordenando la información para ser presentada a sus compañeros. En este quinto y último encuentro, se trabajó conjuntamente en la elaboración de una presentación "power point" que reuniera los datos más relevantes del proyecto de investigación, sintetizando lo estudiado durante este período de tiempo. El producto logrado fue el siguiente: 


\section{FASE III - PRODUCTO FINAL}

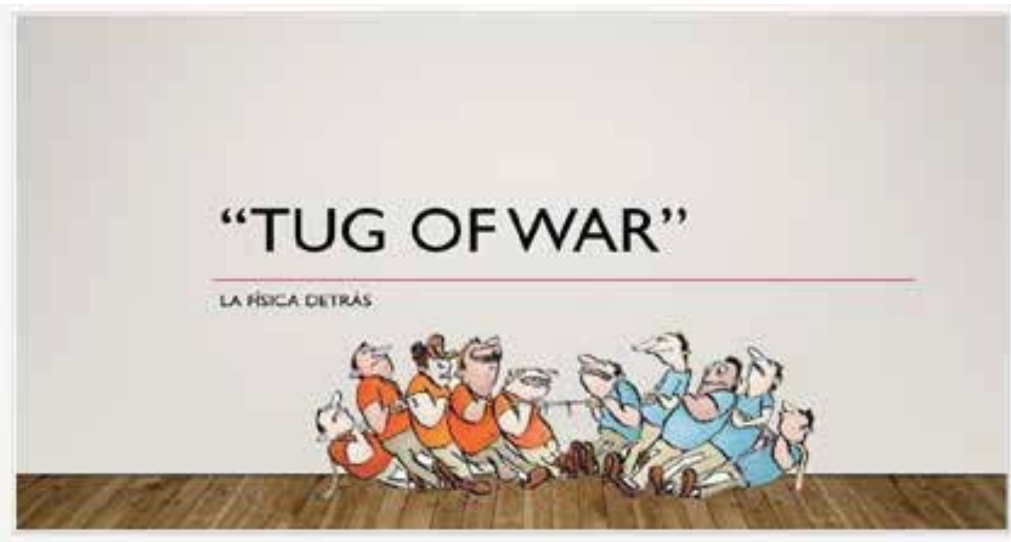

Figura 12: Presentación elaborada por los alumnos

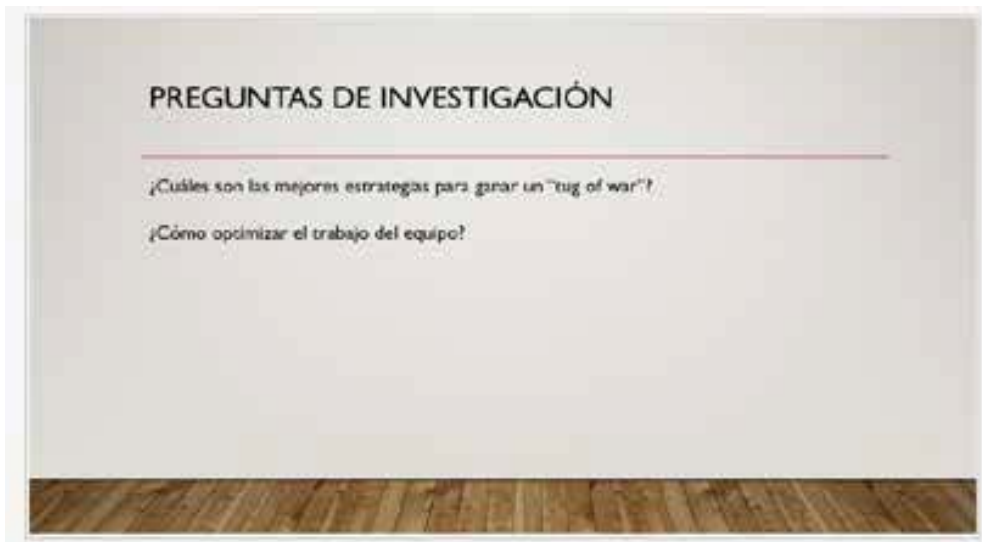

Figura 13: Presentación de las preguntas de investigación

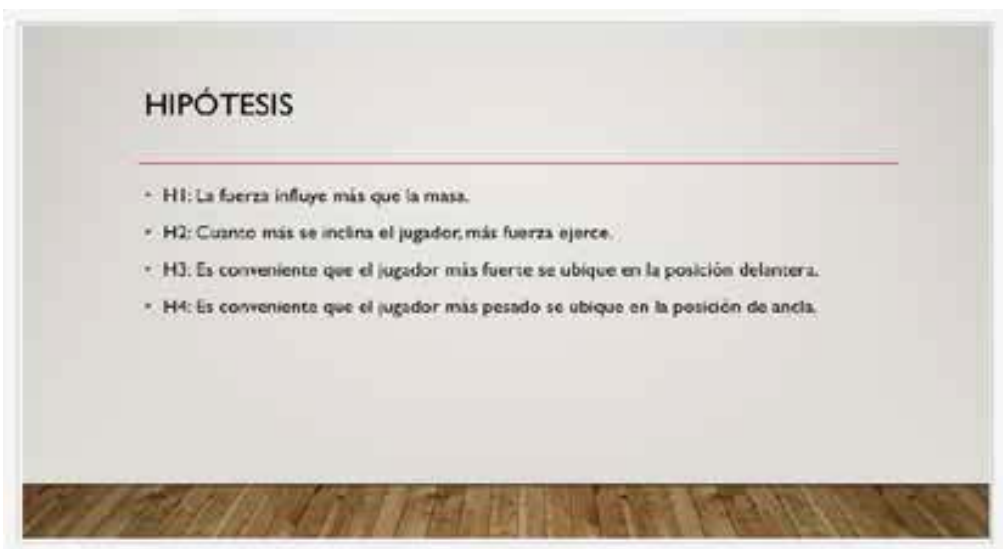

Figura 14: Presentación de las hipótesis de investigación 


\section{HI RECHAZADA}

- En un "taz of war" con solo dos jugadores en el miumo terreno y con la misma postura. quien gass es aquel que siene mayor mas y por lo tanto geners mapor fuerza de friccion y menor aceleración (2ds ley de Newton).

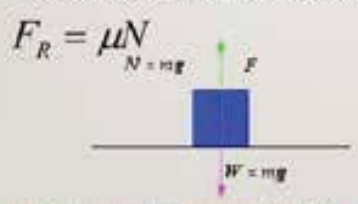

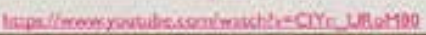

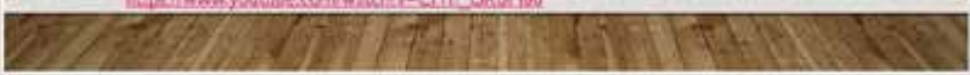

Figura 15: Presentación de la hipótesis 1

\section{H2 ACEPTADA}

- La fuera de craccion cambia según la posturs del cuerpo del ugzdoc y aumenaa en 28 K8 por cadu grado gee esscr dieninuye.

- La maxima fuera de traccion es ejercida con una inclación del cuerpo de los jugadores de entre $35^{\circ}$ y $45^{\circ}$ respecto a ta superfioe.

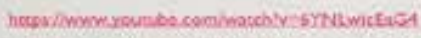

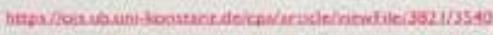

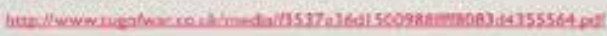

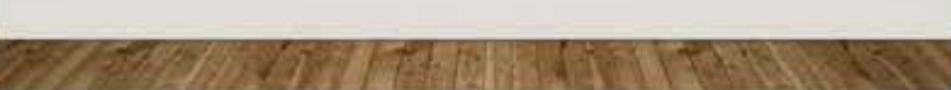

Figura 16: Presentación de la hipótesis 2

\section{H3 RECHAZADA}

- La porsona mis therte debe povicionarse al finsl pars que pueda velitrar mejor su tuorza y teng menos probabcidades de resbilarse.

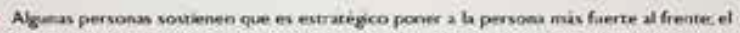
problema de hacerlo es que sil la pericns cae, se pierse al jopidor mis importante.

- Se puede solocar a la periena nibis fuerte al fircil y a ouro jupador fuerse al frence. En teneral se debe meaciar a los vezidores mis fuertes con los mas debiles para que no ehis una fis con solo vegidorer debiles que afecten de forma negativa sl equipa

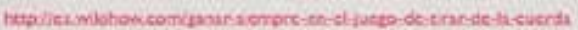

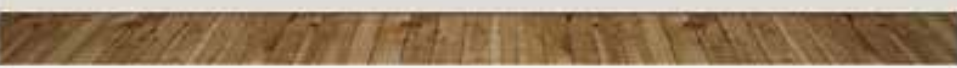

Figura 17: Presentación de la hipótesis 3 


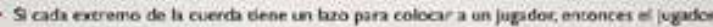

mas fueres debe ir an el laxo.

- Dado cue a tuera que puedo ejercer una penons et directarsente proportional a bu

masa, ye una de la persona mís pesada.

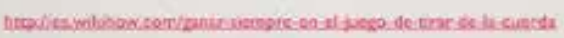

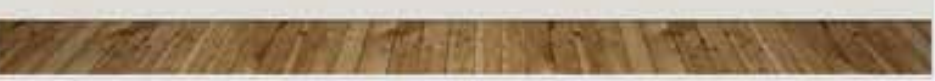

Figura 18: Presentación de la hipótesis 4

\section{CONSIDERACIONES FINALES}

Tal como se percibe en los ejemplos presentados en este artículo, las actividades de enriquecimiento tipo III son una metodología de trabajo que tanto estudiantes como docentes disfrutan. Es una forma de acercar a los estudiantes a la investigación y darles riendas sueltas para que ellos mismos puedan guiar su propio proyecto, de acuerdo a sus intereses y nivel de profundidad que deseen. El rol del docente como facilitador, le proporciona a los estudiantes la libertad de manejar los tiempos y los recursos según los necesite y organizarse en su propio aprendizaje. Los docentes que se han capacitado mediante las formaciones en Altas Habilidades/Superdotación durante los años 2014 - 2016 comentan lo fructífero de la experiencia y la necesidad que todos los docentes puedan capacitarse en este tópico. Estos han cambiado su forma de posicionarse en el aula frente a sus estudiantes en general y en particular han aprendido a identificar y atender las necesidades educativas dentro del aula a aquellos que poseen AH/SD.

Mediante el trabajo de investigación desarrollado en la escuela rural por María, ésta pudo expresar sus intereses académicos y asociarlos a sus vivencias y experiencias de niña rural, pudiendo expandir sus horizontes de aprendizaje y potenciar las altas habilidades que posee en las áreas lingüística, lógico matemática, espacial, naturalista e interpersonal fundamentalmente.

El formato de trabajo abordado en la oportunidad permitió avances conceptuales y procedimentales de muy buena calidad; aumentó considerablemente la autoestima de la niña participante y abrió un sin fin de posibilidades y de profundización académica a nivel de contenidos y por ende de aprendizajes y experiencias.

Desde el punto de vista de la estudiante esta experiencia de investigación constituyó un hito fundamental desde su metodología de estudio, debido a que abrió un abanico de estrategias que permitieron mejorar su capacidad de acercamiento a los conocimientos, procesamiento y organización de los mismos, evidenciándose procesos de aprendizaje cada vez más avanzados.

Se observó una mayor maduración de los procesos de adquisición y manejo disciplinar además de una mayor capacidad de autorregulación y conocimiento de las formas de aprendizaje personales; elementos fundamentales para un desarrollo adecuado de las potencialidades de la estudiante desde lo curricular, pero fundamentalmente desde el punto de vista personal. Se considera fundamental que los estudiantes con Altas Habilidades sean conscientes de sus potencialidades y puedan aprovecharlas para mejorar sus desempeños en todos los niveles. Estos procesos personales llevan un tiempo importante de conocimiento y autoevaluación que mediante la aplicación de esta metodología de trabajo el estudiante posee tiempos personales para llevarlos a cabo y madurar las experiencias y conocimientos adquiridos o experimentados.

El formato de trabajo en proyecto de investigación permitió que su familia se involucrara en las actividades académicas de la niña, así como llevar la labor de investigación más allá de las fronteras físicas de la escuela, permitiendo un trabajo de campo arduo y prolongado, así como la interrelación y colaboración de vecinos de la institución que se interesaron y colaboraron (no solo con la estudiante), sino con la institución en ge- 
neral donando material de estudio. Esto demuestra una mayor toma de conciencia acerca de la necesidad de apoyar los alumnos con Altas Habilidades, no solo desde el ámbito familiar de la estudiante, sino desde la comunidad educativa en sí misma, que vió beneplácito la experiencia desarrollada en la institución educativa.

La instancia de armado de un producto final (exposición y visita guiada) fue muy enriquecedora desde lo conceptual, procedimental y actitudinal; en el entendido que la estudiante debió poner en juego los conocimientos trabajados con anterioridad, los adquiridos durante la investigación y su posterior organización para exponerlos a un público determinado. Es importante destacar que la muestra o exposición continuó creciendo en número y variedad de piezas hasta la actualidad que funciona como museo natural abierto a otras escuelas rurales.

En el caso de los adolescentes del liceo, se trata de alumnos que poseen una diversidad de intereses que abarcan distintas áreas: Matemática, Física, Química, Educación Física, Economía y Sociología. Analizando el tema seleccionado por estos alumnos, tiene sentido que hayan elegido investigar "La física detrás de un tug of war", dado que se trata de una realidad cercana a ellos. Por un lado, en el curso de Física de tercer año de secundaria, se estudia principalmente las diferentes fuerzas que actúan sobre un objeto, como se pudo apreciar en el listado de conocimientos previos que elaboraron los alumnos. Por otro lado, todos los años, se dedica un día entero en su institución educativa a la realización de deportes y entre ellos se destaca la competencia de equipos, dentro de cada generación de alumnos, en el "tug of war". El vínculo existente entre el tema elegido por los estudiantes y su experiencia se identificó como un verdadero facilitador del proceso.

Respecto a la elección de la pregunta central, “¿Cuáles son las mejores estrategias para ganar un tug of war?", pudo estar motivada por un tipo de problemas que el equipo acostumbra a resolver semanalmente en el espacio de Olimpíadas de Matemática, que consiste en hallar la estrategia ganadora ante un juego independientemente de las jugadas que pueda efectuar el contrincante. El hecho de que los estudiantes no conocieran a priori la respuesta a la pregunta que habían formulado fue, sin duda, un factor de entusiasmo.

La física detrás de un "tug of war" es bastante intuitiva. Sin embargo, el deseo de no equivocarse al exponer las hipótesis respecto a la pregunta central de investigación, condujo a los estudiantes a la experimentación, la cual facilitó el proceso y lo hizo más divertido.

Los cuatro alumnos demostraron estar interesados y motivados durante el trabajo de campo, pero se identificó como dificultad la pereza que sintieron al momento de documentar lo investigado, por lo que hallaron beneficioso para el equipo, incorporar en futuras instancias a estudiantes con otras habilidades tales como la comunicación, el diseño y las relaciones interpersonales entre otras. Sin embargo, mas no redactaron un documento para entregar, pero sí se esforzaron por armar una presentación clara que les sirviera como soporte para mostrarles los hallazgos a sus compañeros de clase y he aquí la importancia de contar con una audiencia.

Cabe destacar que esta experiencia se tradujo en una oportunidad de aprendizaje del método científico, si bien no se quiso complejizar el proceso de investigación introduciendo conceptos tales como hipótesis nulas e hipótesis alternativas. Los estudiantes entendieron en profundidad el método -pues lo pusieron en práctica- y quedaron preparados para utilizarlo con autonomía ante futuras interrogantes que se planteen. Asimismo, la experiencia permitió la sinergia entre los integrantes del equipo, la cual potenció sus habilidades socioemocionales y cognitivas.

En suma, la concepción de los tres anillos de Renzulli concibe los comportamientos superdotados en la intersección de a) habilidad por encima del promedio, b) creatividad y c) compromiso con la tarea. Las actividades de enriquecimiento tipo III son una herramienta que facilita el desarrollo de estos comportamientos y prepara a los niños para ser los futuros productores de conocimiento y nuevos medios de comunicación del futuro. Los ejemplos aquí compartidos son uno de los tantos que se han llevado a cabo en instituciones educativas de Uruguay con estudiantes de primaria y secundaria con Altas Habilidades/Superdotación. Los docentes formados en estas instancias de capacitación continúan identificando y trabajando en pro de ofrecer servicios especializados y orientados a atender las necesidades educativas y sociales de los estudiantes con AH/SD de Uruguay. 


\section{REFERENCIAS}

Austrialian Curriculum, Assessment and Reporting Authority (ACARA). (2013). The Australian curriculum: Mathematics. Retrieved from:

http://www.australiancurriculum.edu.au/Mathematics/Content-structure.

Bendelman, K (2012). Jugando con el futuro: Estimulando talentos a través de la creación de videojuegos. Revista ldeacción, 32 (1), 180-195.

Bendelman, K \& Pérez, S. G (2016). Altas Habilidades/Superdotación. ¿Qué, quién, cómo?. Montevideo, Uruguay: Isadora Ediciones.

Garcia-Cepero, M. C. (2008). The Enrichment Triad Model: nurturing creative-apa productivity among college students. Innovations in Education and Teaching International, 45(3), 295-302. https://doi.org/10.1080/14703290802176238

Katz, L.G., S.C. Chard, \&Y. Kogen. 2014. Engaging Children's Minds: The Project Approach. 3rd ed. Santa Barbara, CA: Praeger.

Maker, C.J., \& Schiever, S.W. (2010). Curriculum development and teaching strategies for gifted learners (3rd ed.). Austin, TX: Pro-Ed.

Postholm, M. B. (2015). Classroom Management in Project Work. Creative Education, 6, 2077-2089. http://dx.doi. org/10.4236/ce.2015.619212

Renzulli, J.S., \& Reis, S. M. (1997). The Schoolwide Enrichment Model. A how- to guide for educational excellence. (2nd Ed.). Mansfield Center, CT: Creative Learning Press, Inc.

Reis, S. M., \& Renzulli, J. S. (2004). Current research on the social and emotional development of gifted and talented students: Good news and future possibilities. Psychology in the Schools, 41(1), 119-130. https://doi.org/10.1002/ pits. 10144

Renzulli, J. S. (1978). What makes giftedness? Reexamining a definition. Phi Delta Kappan, 60, 180-184, 261. https://doi. org/10.1177/003172171109200821

Renzulli, J. S. (1995). Teachers as Talent Scouts. Educational Leadership, 52(4), 75-81.

Renzulli, J. S. (2016). Short Three-ring definition. Document e-mailed to Pérez Barrera, S. on May, 3, 2016.

Stepanek, J. (1999). The Inclusive Classroom. Meeting the Needs of Gifted Students: Differentiating Mathematics and Science Instruction. It $\Downarrow$ s just good teaching, 1-50. http://www.nwrel.org/msec.

Tomlinson, C.A., (1999). The differentiated classroom: Responding to the needs of all learners. Alexandria, VA: Association for Supervision and Curriculum Development.

Renzulli, J. (2014). Uconn Talks. Recuperado de: https://youtu.be/g_hPhKvduts

United Nations Organization for Education, Science and Culture [UNESCO], (2017). La UNESCO avanza. La agenda 2030 para el desarrollo sostenible. París, Francia.

Villalobos, X. (2011). Reflexión en torno a la gestión de aula y a la mejora en los procesos de enseñanza y aprendizajes. Revista Iberoamericana de Educación, 1-7.

Zmood, S. (2014). Fostering the promise of high achieving mathematics students through curriculum differentiation. In Curriculum in focus: Research guided practice. (Proceedings of the 37th annual conference of the Mathematics Education Research Group of Australasia) (pp 677-684).

Fecha Recepción: 03/09/2018

Fecha Aceptación: 26/11/2018 